\title{
Zona pellucida resistance to sperm penetration before the completion of human oocyte maturation
}

\author{
J. Tesařík, L. Pilka and P. Trávník* \\ Laboratory of Human Reproduction, First Department of Obstetrics and Gynaecology, and \\ *Department of Histology and Embryology, Faculty of Medicine, J. E. Purkyně University, Brno, \\ Czechoslovakia
}

\begin{abstract}
Summary. Human oocytes exposed to capacitated spermatozoa in vitro when at metaphase of the lst meiotic division (metaphase I) were not penetrated, even though some subsequently progressed to metaphase of the 2 nd meiotic division (metaphase II). When the non-penetrated oocytes that had reached metaphase II during the incubation with spermatozoa were freed from the zona pellucida and reinseminated, two or more pronuclei developed in most of them. By contrast, no penetration was observed when the oocytes were reinseminated in the zona-intact state. When compared with metaphase II oocytes, metaphase I oocytes had a similar zona-binding capacity for spermatozoa, but fewer spermatozoa were found within the zona. These results indicate that the zona pellucida of human oocytes undergoes important maturational changes during the transition from metaphase I to metaphase II. Ultrastructural and previous histochemical findings suggest that these changes involve secretions from both the oocyte and cumulus cells and that the increased zona resistance at metaphase I may be due to relative insufficiency of cumulus cell-secreted 'softening' factors. If the integrity of the cumulus oophorus is disturbed at this stage, this condition becomes irreversible.
\end{abstract}

Keywords: zona pellucida maturation; sperm-egg interaction; oocyte meiosis; cumulus cells; human

\section{Introduction}

The reaction of spermatozoa with the zona pellucida is a key event regulating their access to the oocyte at fertilization. Under physiological conditions the zona is responsible for homologous gamete recognition and binding (Hartmann, 1983) and it also plays a major role in the mechanism that prevents polyspermy (Wolf, 1981; Sathananthan \& Trounson, 1982). On the other hand, the development of increased zona resistance to sperm penetration, leading to decreased oocyte fertilizability, has been described in various experimental systems, such as during maturation of cumulus-free mouse oocytes in vitro (De Felici \& Siracusa, 1982) or in mouse oocytes aged in vivo or in vitro (Austin, 1970; Szöllösi, 1975; Longo, 1981; Gianfortoni \& Gulyas, 1985). Impairment of sperm-zona interaction has also been suggested to be a contributing factor to idiopathic human infertility (Trounson et al., 1980; Mahadevan et al., 1983). For a better understanding of the mechanisms underlying these disorders more information about the zona alterations during the final phase of oocyte meiotic maturation is obviously needed. Based on circumstantial evidence (Trounson et al., 1982; Zenzes et al., 1985), the zona pellucida of human oocytes approaching meiotic maturity seems to undergo some maturational changes which render it more susceptible to sperm penetration. Incomplete oocyte maturation was found to be the main course of fertilization failure in our in-vitro fertilization programme (J. Tesařik, unpublished data). These observations led us to investigate the relationship between the meiotic status of human oocytes and the penetrability of the zona pellucida. 


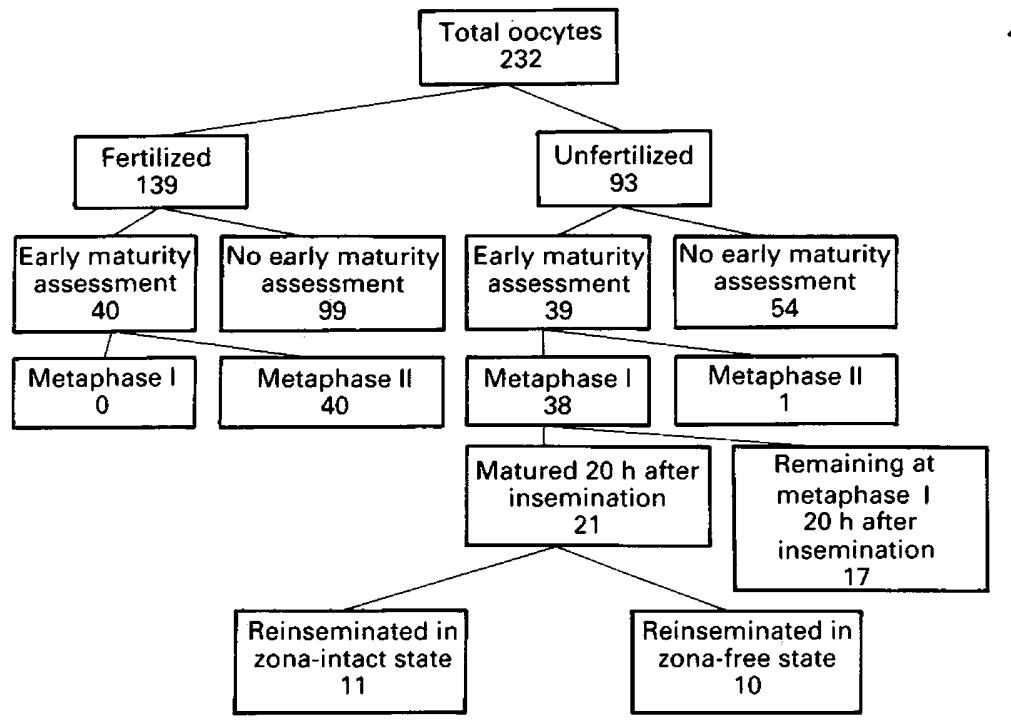

Fig. 1. Schematic representation of the allocation of oocytes under study to different examinations.

\section{Materials and Methods}

This study is based on investigation of 232 in-vitro inseminated oocytes from an in-vitro fertilization programme. The oocytes were subjected to non-destructive microscopic examinations at different times after in-vitro insemination and fertilization results and oocyte maturity status were recorded. Oocytes possessing two pronuclei and two polar bodies $20 \mathrm{~h}$ after insemination were cultured further and reimplanted into the patient's uterine cavity at the 2- to 8-cell stages of preimplantation development. Some of the unfertilized oocytes that had been at metaphase I at the time of insemination and that had reached metaphase II during the incubation with spermatozoa were reinseminated, either in the zona-intact state or after removal of the zona pellucida, in order to assess the contribution of the zona to the fertilization failure. The utilization of in-vitro inseminated oocytes for different examinations is schematically represented in Fig. 1. Another 15 oocytes ( 7 at metaphase I and 8 at metaphase II) from couples in which the husband was not able to produce a semen sample or when oocyte recovery was performed purely for diagnostic purposes (Pilka et al., 1983) were processed for electron microscopy immediately after recovery.

Collection and handling of gametes. Oocytes were recovered by puncture and aspiration of ovarian follicles under laparoscopic or ultrasonic guidance in patients suffering from tubal or unexplained infertility. Follicular development was stimulated with clomiphene citrate (Gravosan: Spofa, Prague, Czechoslovakia), $100 \mathrm{mg}$ daily on Days 3-7 of the menstrual cycle, which was supplemented, in some cases, with $150 \mathrm{iu}$. human menopausal gonadotrophin (hMG; Pergonal, Serono Laboratories, Inc., Randolph, MA, U.S.A.) administered on Days 6, 8, 10 and 11 of the cycle (Day 1 = first day of menstruation). The clomiphene or clomiphene/hMG treatment was followed by $6000 \mathrm{i}$.u. human chorionic gonadotrophin (hCG; Praedyn; Spofa) injected intramuscularly on the day when the leading follicle had reached $18 \mathrm{~mm}$ in diameter. Follicular aspiration was carried out $34-36 \mathrm{~h}$ after the injection of hCG. Oocytes were identified in follicular aspirates, transferred into plastic test tubes containing I ml modified Ham's F 10 medium (see below) and cultured for 5-8 $\mathrm{h}$ before addition of spermatozoa.

Sperm samples were obtained from apparently healthy partners of the women attending our in-vitro fertilization programme. Only samples which contained $\geqslant 70 \times 10^{6}$ spermatozoa $/ \mathrm{ml}$ semen, $\geqslant 50 \%$ motile spermatozoa, and $\geqslant 70 \%$ normal sperm forms were included in this study. Spermatozoa were washed from seminal plasma and capacitated in vitro for $5-6 \mathrm{~h}$ as described previously (Tesařík et al., 1984).

Culture conditions and gamete interaction. Ham's F 10 medium (Ham, 1963) was modified by the addition of $0.5 \mathrm{~g}$ calcium lactate/l (Serva Feinbiochemica, Heidelberg, F.R.G.), 0.25 g sodium pyruvate/l (Merck, Darmstadt, F.R.G.), and $1.3 \mathrm{~g}$ sodium bicarbonate/l (Serva). These compounds were added to Ham's $F 10$ dried powder (Flow Laboratories, Rockville, MD, U.S.A.) together with 25000 i.u. penicillin/l and $0.04 \mathrm{~g}$ streptomycin/l (both purchased from Serva) and all components were dissolved in four-times glass-distilled water to a final osmotic pressure of $285 \mathrm{mosmol} / \mathrm{kg}$ on the day before use of the medium. The medium was enriched by $16 \%(\mathrm{v} / \mathrm{v})$ heat-inactivated serum from the patient and 
equilibrated with a gas phase of $90 \% \mathrm{~N}_{2}, 5 \% \mathrm{O}_{2}$ and $5 \% \mathrm{CO}_{2}$. This medium was used for all cultures and incubations including oocyte pre-insemination culture, inseminations and embryo cultures.

Insemination in vitro was carried out 5-8 h after oocyte recovery by adding $1 \times 10^{5}$ spermatozoa from capacitation cultures to $1 \mathrm{ml}$ medium containing the oocyte. Most oocytes were left undisturbed in this fertilization culture until the following day when fertilization results were assessed (see below). Oocytes were sometimes removed from the fertilization culture $1 \mathrm{~h}$ after insemination, remnants of cumulus oophorus and corona radiata cells were separated from the oocytes under a dissecting microscope, and the oocytes were examined for the presence of the 1st polar body. The duration of the whole procedure did not usually exceed $1 \mathrm{~min}$; the oocytes were then returned immediately to the fertilization culture.

At $24 \mathrm{~h}$ after insemination all remaining corona cells and loosely adhering spermatozoa were removed through repeated aspirations into finely drawn Pasteur pipettes and oocytes were examined, in an inverted microscope equipped with Nomarski optics, for the presence of pronuclei and polar bodies. Reinsemination of unfertilized oocytes was performed using sperm suspensions newly prepared in the same way as for the first insemination and fertilization results were checked after an additional $16-18 \mathrm{~h}$.

Preparation and insemination of zona-free oocytes. Some of the unfertilized oocytes that lacked the 1st polar body i h after insemination but progressed to metaphase II during the incubation with spermatozoa (assessed $20 \mathrm{~h}$ after insemination) were incubated for $5 \mathrm{~min}$ in $0.1 \%(\mathrm{w} / \mathrm{w})$ pronase (Sigma Chemical Co., St Louis, MO, U.S.A.) dissolved in serum-free F 10 medium. The oocytes were then transferred into F 10 medium supplemented with $16 \%$ heatinactivated human serum and the partly digested zonae pellucidae were removed by repeated aspirations into fine Pasteur pipettes. After a thorough washing in the medium the zona-free oocytes were reinseminated in vitro with $1 \times 10^{5}$ spermatozoa $/ \mathrm{ml}$ and examined for the presence of pronuclei $16-18 \mathrm{~h}$ later.

Electron microscopy. Oocytes ( 15 freshly recovered and 41 inseminated) were fixed in $3 \%$ glutaraldehyde buffered with $0.1 \mathrm{M}$-cacodylate (pH 7.2), post-fixed with $1 \%$ osmium tetroxide in the same buffer, dehydrated in ethyl alcohol and acetone, and embedded in Durcupan ACM. Alternate series of thick $(1 \mu \mathrm{m})$ and thin sections $(\sim 80 \mathrm{~nm})$ were cut with an Ultrotome III LKB ultramicrotome. The thick sections were stained with Azur A and examined in the light microscope; the thin sections were stained with aqueous uranyl acetate and Reynold's lead citrate, and viewed with a Tesla BS 500 electron microscope.

Quantitation of sperm-zona pellucida interaction. In 35 living oocytes the number of zona-associated spermatozoa was determined when the oocytes were examined for the presence of pronuclei. The spermatozoa were counted in all focal planes with a $\times 40$ objective. The relative proportions of spermatozoa bound to the zona surface and those penetrating into the depth of this oocyte vestment were assessed in thin sections prepared from 25 oocytes of this group (17 at metaphase I and 8 at metaphase II) and examined in the electron microscope. Three to four section planes of each oocyte (those in which most spermatozoa were evident) were chosen for sperm counting. These counts usually totalled $40-50 \%$ of the oocyte-associated spermatozoa. The significance of differences in the number of zona pellucida-associated spermatozoa between individual types of oocytes was assessed by $t$ tests.

\section{Results}

\section{Retrospective analysis of oocyte maturity status as related to its fertilizability}

When the fertilization results, as evaluated $20 \mathrm{~h}$ after insemination, were related to the actual number of polar bodies in individual oocytes (Table 1), it was evident that the absence of polar bodies was the most frequent finding in unfertilized oocytes. Of these metaphase I oocytes, 8 were examined by electron microscopy and no sperm components could be identified in the ooplasm or perivitelline space. One polar body was observed in $45 \%$ of unfertilized oocytes, while the presence of 2 polar bodies was an exceptional finding (probably resulting from division of the lst polar body).

\section{Early evaluation of oocyte maturity status as related to its fertilizability}

When the fertilization outcome (assessed $20 \mathrm{~h}$ after insemination) was related to the oocyte maturity status $1 \mathrm{~h}$ after insemination, no metaphase I oocyte became fertilized, while as many as $98 \%$ of metaphase II oocytes developed two pronuclei (Table 2). However, 21 of 38 metaphase I oocytes reached metaphase $I 1$ within $20 \mathrm{~h}$ after insemination. These findings indicate that many of the unfertilized oocytes showing one polar body $20 \mathrm{~h}$ after insemination (see Table 1) must have entered metaphase II only in the fertilization culture, after the addition of spermatozoa. 
Table 1. Relationship of the actual number of polar bodies to fertilization outcomes for human oocytes $20 \mathrm{~h}$ after in-vitro insemination

\begin{tabular}{|c|c|c|c|c|c|c|c|}
\hline \multirow{3}{*}{$\begin{array}{l}\text { Fertilization } \\
\text { outcome* }\end{array}$} & \multirow{3}{*}{$\begin{array}{c}\text { Total } \\
\text { oocytes }\end{array}$} & \multicolumn{6}{|c|}{$\begin{array}{l}\text { Oocytes with different nos. } \\
\text { of polar bodies } \dagger\end{array}$} \\
\hline & & \multicolumn{2}{|c|}{0} & \multicolumn{2}{|c|}{1} & \multicolumn{2}{|c|}{2} \\
\hline & & No. & $\%$ & No. & $\%$ & No. & $\%$ \\
\hline Fertilized & 139 & - & - & - & - & 139 & 100 \\
\hline Unfertilized & 93 & 50 & 54 & 42 & 45 & 1 & 1 \\
\hline
\end{tabular}

*An oocyte was considered fertilized if there were two pronuclei in the ooplasm $20 \mathrm{~h}$ after insemination; it was considered unfertilized if no nuclear structure was visible.

†Examined $20 \mathrm{~h}$ after insemination.

Table 2. Maturity status of fertilizable and unfertilizable human oocytes $1 \mathrm{~h}$ after in-vitro insemination

\begin{tabular}{lcrrrrrr}
\hline \multirow{2}{*}{$\begin{array}{l}\text { Maturity } \\
\text { status* }\end{array}$} & Total & \multicolumn{2}{c}{ Fertilized $\dagger$} & & \multicolumn{2}{c}{ Unfertilized $\dagger$} \\
\cline { 3 - 4 } \cline { 7 - 8 } & oocytes & No. & $\%$ & & No. & $\%$ \\
\hline Metaphase I & 38 & 0 & 0 & & 38 & 100 \\
Metaphase II & 41 & 40 & 98 & & 1 & 2 \\
\hline
\end{tabular}

*Examined $1 \mathrm{~h}$ after in-vitro insemination.

$\dagger$ Evaluated $20 \mathrm{~h}$ after insemination (see * footnote in Table 1).

Table 3. Penetration results in metaphase I human oocytes matured to metaphase II in the fertilization culture and reinseminated in the zona-intact or zona-free state

\begin{tabular}{lcrlll}
\hline & & \multicolumn{5}{c}{ No. of pronuclei* } \\
\cline { 3 - 6 } $\begin{array}{l}\text { Zona } \\
\text { pellucida }\end{array}$ & $\begin{array}{c}\text { Total } \\
\text { oocytes }\end{array}$ & 0 & 1 & 2 & $>2$ \\
\hline Present & 11 & 10 & 1 & 0 & 0 \\
Absent & 10 & 1 & 0 & 1 & 8 \\
\hline
\end{tabular}

*Examined $16-18 \mathrm{~h}$ after reinsemination.

Values are numbers of eggs showing the numbers of pronuclei indicated.

\section{Reinsemination of zona-intact and zona-free oocytes}

Of the 21 metaphase I oocytes that reached metaphase II only after insemination (see above), 11 oocytes were reinseminated in the zona-intact state and the other 10 oocytes were reinseminated after removal of the zona. At least 2 pronuclei developed in 9 oocytes of the latter group, while no penetration occurred in the former (Table 3). At the time of assessment (16-18 h after reinsemination) most of the unpenetrated zona-intact oocytes still possessed condensed metaphase II 

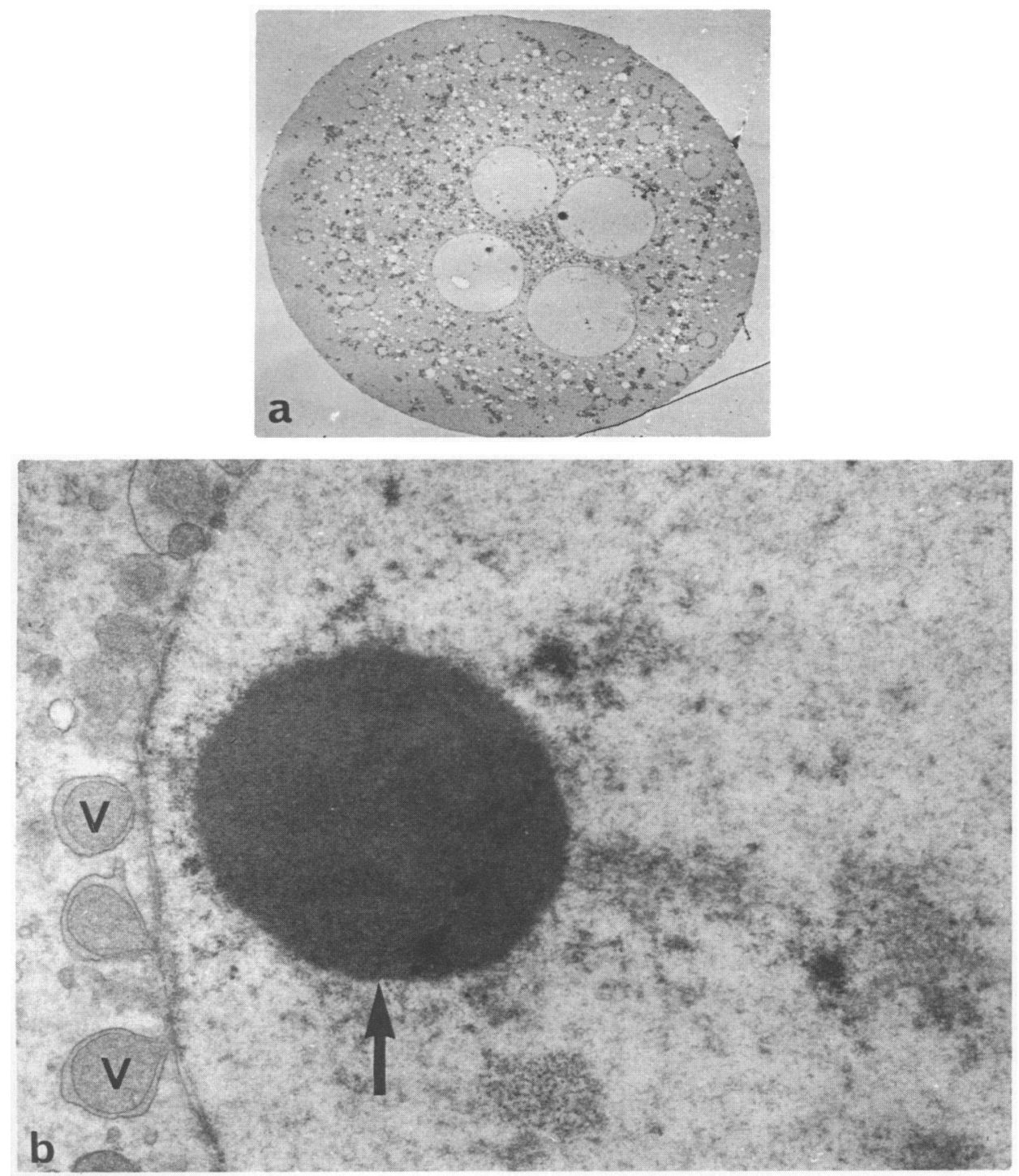

Fig. 2. Metaphase I human oocyte having matured to metaphase II in the insemination culture and reinseminated in the zona-free state after the failure of the first insemination. (a) Equatorial section of the oocyte showing 4 pronuclei. $\times 700$. (b) Normally structured chromatin and nucleolus-like body (arrow) are seen at a higher magnification. Vesicles (V) within dilated segments of the nuclear envelope are also typical structures of human pronuclei. $\times 30000$.

chromosomes. In only one case had the female pronucleus developed and a second polar body separated. Ultrastructural examination of the zona-free human oocytes penetrated by spermatozoa in vitro (Fig. 2) did not demonstrate any differences in the appearance of individual pronuclei compared to presumably normal human pronuclear eggs (Tesařík \& Dvořák, 1981). The pronuclei had the normal size and shape (Fig. 2a), showed the typical structure of chromatin and the nuclear envelope and possessed nucleolus-like bodies (Fig. 2b). Profiles of the axonema and remnants of other sperm tail structures were usually seen close to the pronuclei. 
Table 4. Sperm-zona pellucida association $20 \mathrm{~h}$ after in-vitro insemination in relation to human oocyte maturity status assessed $1 \mathrm{~h}$ after insemination

\begin{tabular}{lcccccc}
\hline \multirow{2}{*}{$\begin{array}{l}\text { Maturity } \\
\text { status }\end{array}$} & \multicolumn{2}{c}{ Total oocytes } & & \multicolumn{3}{c}{ Zona-associated spermatozoa } \\
\cline { 2 - 3 } \cline { 7 - 8 } & Fertilized & Unfertilized & & No./oocyte* & \% surface-bound & $\%$ penetrated $\dagger$ \\
\hline Metaphase I & 0 & 17 & & $52 \cdot 5 \pm 18 \cdot 4$ & $92 \ddagger$ & $8 \ddagger$ \\
Metaphase II & 17 & 1 & & $61 \cdot 0 \pm 19 \cdot 3$ & $73 \S$ & $27 \S$ \\
\hline
\end{tabular}

*Values are mean \pm s.d.

† A spermatozoon was considered 'penetrated' when the sperm head was totally within the zona pellucida.

\$All metaphase I oocytes were examined by electron microscopy for the evaluation of sperm-zona association.

$\S$ Determined in 8 oocytes of this group which were examined by electron microscopy.

\section{Relationship between oocyte maturity status and sperm-zona interaction}

All of the metaphase I oocytes used in this experiment failed to become fertilized, while fertilization was successful in most of the metaphase II oocytes. Even though the mean number of spermatozoa associated with the zona was slightly lower in the former group of oocytes (Table 4), this difference was not significant. There were remarkable differences in the number of zona-associated spermatozoa between individual oocytes within both groups. On the other hand, the ultrastructural analysis revealed a significantly higher percentage of spermatozoa having penetrated into the zona pellucida of metaphase II oocytes as compared to metaphase I oocytes in which a great majority of zona-associated spermatozoa were surface-bound (Table 4).

\section{Structural changes in the zona pellucida during final phases of oocyte maturation}

Marked differences were noticed in the ultrastructure of the zona pellucida between metaphase I and metaphase II oocytes fixed immediately upon recovery (Fig. 3). The zona of metaphase I oocytes gave an impression of a rather compact and homogeneous layer distinctly demarcated against the cumulus oophorus, showing only little intercellular material demonstrable by electron microscopy (Fig. 3a). By contrast, the zona of metaphase II oocytes displayed a highly porous structure in its outer one-third, showed wide slits filled with the newly formed abundant cumulusoophorus intercellular material at its surface and was in general poorly delineated towards the fully expanded cumulus the intercellular matrix of which penetrated deep into its outer part (Fig. 3b). The oocyte showed a noticeable secretory activity as early as metaphase I, as judged by the presence of numerous secretory granules in the cortical cytoplasm with many granules in the course of exocytosis releasing their contents to the perivitelline space (Fig. 4) where deposition of glycoproteins secreted by preovulatory human oocytes has previously been described (Tesařik \& Kopečný, 1986). The granules undergoing exocytosis had the same size but a slightly lower electron density than the typical cortical granules. No apparent changes in this secretory activity were noted between metaphase I and metaphase II oocytes.

\section{Discussion}

We have shown that human oocytes inseminated in vitro before they have completed their meiotic maturation fail to be fertilized in our in-vitro fertilization system and that this failure is largely due to the inability of spermatozoa to penetrate the zona pellucida. Low fertilization rates have also been reported for human oocytes recorded as immature, based on morphological assessment of cumulus and corona cells (Zenzes et al., 1985). In contrast to our results, penetration was achieved, although at relatively low rates, in human metaphase $\mathbf{I}$ oocytes incubated with higher concentrations of spermatozoa $\left(0 \cdot 5-1.0 \times 10^{6} / \mathrm{ml}\right.$, i.e. 5-10 times more than in this study) 

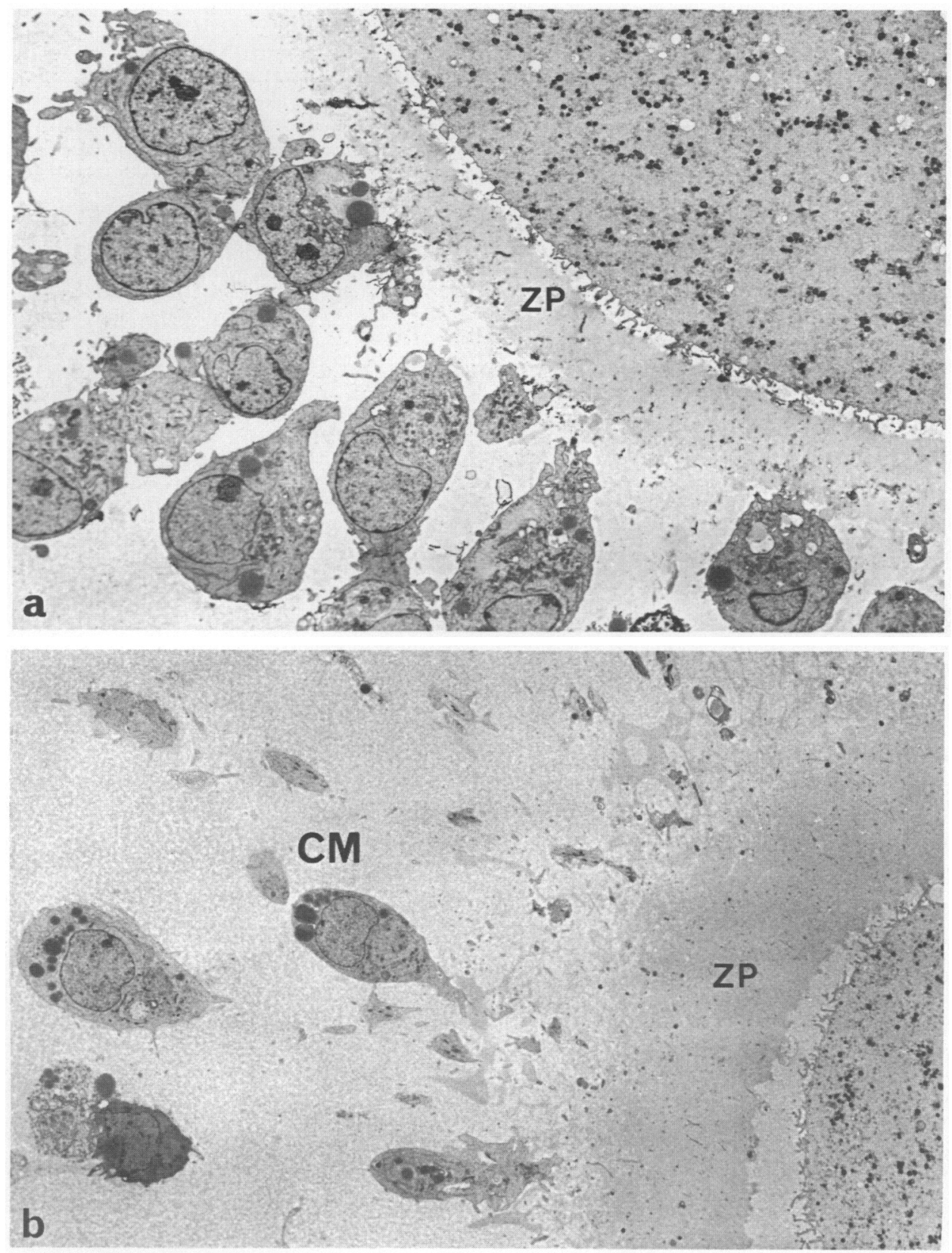

Fig. 3. Structural differences between the zona pellucida (ZP) surrounding a metaphase I (a) and metaphase II (b) human oocyte. Note the porous structure of the outer third of the zona in the metaphase II oocyte contrasting with the relatively compact, homogeneous and well demarcated zona of the metaphase I oocyte. CM, cumulus intercellular material. $\times 2000$.

(Nishimoto et al., 1982). By use of elevated sperm concentrations even oocytes at earlier stages of meiosis could be penetrated (Overstreet \& Hembree, 1976; Overstreet et al., 1980). It seems therefore that the increased zona resistance experienced by the oocyte before it has reached full meiotic maturity is a relative rather than an absolute condition which may be overcome by increasing the concentration of spermatozoa in the fertilization culture. 


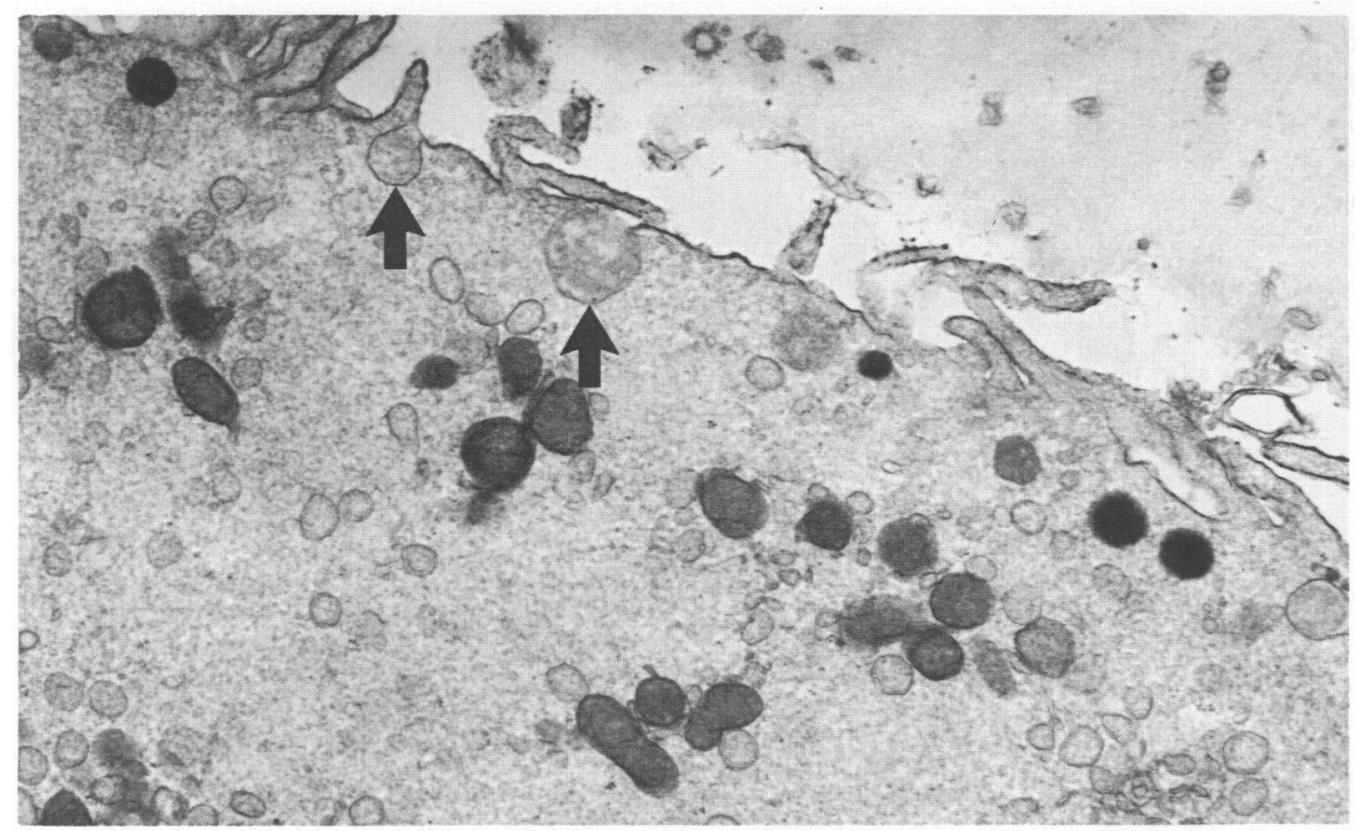

Fig. 4. Cortical cytoplasm of a metaphase I human oocyte showing secretory granules in the course of exocytosis (arrows). $\times 25000$.

Collectively the results of this study strongly suggest that the zona pellucida of human oocytes undergoes important maturational changes during oocyte transition from metaphase I to metaphase II. In fact, autoradiographic and histochemical data indicate an active secretion of proteoglycans from metaphase II human oocytes and their surrounding cumulus cells into the zona pellucida (Tesařík \& Kopečný, 1986). In the present study we observed ultrastructural signs of active secretion from the oocyte even at metaphase $I$ when the cumulus cells had not yet fully activated their intercellular matrix production. This oocyte secretion is apparently a physiological event as signs of spontaneous in-vivo release of granules from the cortical cytoplasm of human follicular oocytes were observed, irrespective of whether the oocytes were obtained in unstimulated or stimulated cycles (Rousseau et al., 1977). The relationship between this spontaneous granule release and the cortical reaction is not clear. It is possible that a continuous slow exocytosis of cortical granules is a contributory factor to the zona pellucida maturation during the final phases of human oocyte meiosis, while the true cortical reaction, in response to the fertilizing spermatozoon, merely represents an abrupt dramatic acceleration of this process.

The impregnation of the outer part of the zona pellucida with cumulus cell-secreted materials, which typically occurs in metaphase II human oocytes (Tesařík \& Kopečný, 1986), is shown here to coincide with a marked increase in the zona penetrability. It is tempting to suggest that the cumulus cells of meiotically mature oocytes contribute to their zona pellucida a 'softening' factor(s) antagonizing the effect of presumed 'hardening' factors secreted by the oocyte during earlier stages of maturation. If the cumulus cells are removed or altered before the achievement of oocyte meiotic maturity, as for premature in-vitro insemination, the cumulus-cell secretions cannot assume this function and the elevated resistance of the zona to spermatozoa becomes irreversible. This may explain why reinsemination in the zona-intact state is inefficient for oocytes having reached metaphase II only during the incubation with spermatozoa, while such oocytes are easily penetrated and develop pronuclei when reinseminated after removal of the zona. Poor fertilization rates after reinsemination that did not differ significantly from the rates of spontaneously delayed fertilization (without reinsemination) have been described by others (Trounson \& Webb, 1984). 
From these results it follows that a proper timing of sperm-egg interaction is of prime importance for the success of in-vitro fertilization in current programmes which in general tend to use lower concentrations of spermatozoa for inseminations. Many in-vitro fertilization failures appear to be due to premature addition of spermatozoa to meiotically immature oocytes. Because human oocytes can be successfully matured in vitro (Edwards et al., 1969; Nishimoto et al., 1982) and pregnancies have been established after transfer of embryos developing from in-vitro matured oocytes (Veeck et al., 1983), the definition of more reliable criteria for oocyte (and zona pellucida) maturity could substantially improve the clinical efficacy of in-vitro fertilization. The search for substances which, added to insemination medium, would help to restore the penetrability of the zona pellucida is another possible way of solving this problem.

We thank Mrs D. Kyjovská for excellent technical assistance.

\section{References}

Austin, C.R. (1970) Ageing and reproduction: postovulatory deterioration of the egg. J. Reprod. Fert., Suppl. 12, 39-53.

De Felici, M. \& Siracusa, G. (1982) Spontaneous hardening of the zona pellucida of mouse oocytes during in vitro culture. Gamete Res. 6, 107-113.

Edwards, R.G., Bavister, B.D. \& Steptoe, P.C. (1969) Early stages of fertilization in vitro of human oocytes matured in vitro. Nature, Lond. 221, 632-635.

Gianfortoni, J.G. \& Gulyas, B.J. (1985) The effects of short-term incubation (aging) of mouse oocytes on in vitro fertilization, zona solubility, and embryonic development. Gamete Res. 11, 59-68.

Ham, R.G. (1963) An improved nutrient solution for diploid chinese hamster and human cell lines. Expl Cell Res. 29, 515-526.

Hartmann, J.F. (1983) Mechanism and Control of Animal Fertilization, pp. 325-363. Academic Press, New York.

Longo, F.J. (1981) Changes in the zonae pellucidae and plasmalemma of aging mouse eggs. Biol. Reprod. 25, 399-411.

Mahadevan, M.M., Trounson, A.O. \& Leeton, J.F. (1983) The relationship of tubal blockage, infertility of unknown cause, suspected male infertility, and endometriosis to success of in vitro fertilization and embryo transfer. Fert. Steril. 40, 755-762.

Nishimoto, T., Yamada, I., Niwa, K., Mori, T., Nishimura, T. \& Iritani, A. (1982) Sperm penetration in vitro of human oocytes matured in a chemically defined medium. J. Reprod. Fert. 64, 115-119.

Overstreet, J.W. \& Hembree, W.C. (1976) Penetration of the zona pellucida of nonliving human oocytes by human spermatozoa in vitro. Fert. Steril. 27, 815-831.

Overstreet, J.W., Yanagimachi, R., Katz, D.F., Hayashi, K. \& Hanson, F.W. (1980) Penetration of human spermatozoa into human zona pellucida and zonafree hamster egg: a study of fertile donors and infertile patients. Fert. Steril. 33, 534-542.

Pilka, L., Trávník, P. \& Tesał̌́k, J. (1983) Diagnose der grundlegenden Sterilitätsursache des Ehepaares mittels einer laparoskopischen Untersuchung. Zentbl. Gynäkol. 105, 487-492.

Rousseau, P., Meda, P., Lecart, C., Haumont, S. \& Ferin, J. (1977) Cortical granule release in human follicular oocytes. Biol. Reprod. 16, 104-111.
Sathananthan, A.H. \& Trounson, A.O. (1982) Ultrastructure of cortical granule release and zona interaction in monospermic and polyspermic human ova fertilized in vitro. Gamete Res. 6, 225-234.

Szölösi, D. (1975) Mammalian eggs aging in the fallopian tubes. In Aging Gametes, pp.98-121. Ed. R.J. Blandau. S. Karger, Basel.

Tesařik, J. \& Dvořák, M. (1981) Ultrastructure of the human ovum fertilized in vitro. Folia morph., Prague 29, 297-304.

Tesał̌ik, J. \& Kopečný, V. (1986) Late preovulatory synthesis of proteoglycans by the human oocyte and cumulus cells and their secretion into the oocyte-cumuluscomplex extracellular matrices. Histochemistry 85, $523-528$.

Tesał̌ik, J., Kopex̌ný, V. \& Dvớák, M. (1984) Selective binding of human cumulus cell-secreted glycoproteins to human spermatozoa during capacitation in vitro. Fert. Steril. 41, 919-925.

Trounson, A.O. \& Webb, J. (1984) Fertilization of human oocytes following reinsemination in vitro. Fert. Steril. 41, 816-819.

Trounson, A.O., Leeton, J.F., Wood, C., Webb, J. \& Kovacs, G. (1980) The investigation of idiopathic infertility by in vitro fertilization. Fert. Steril. 34, 431-438.

Trounson, A.O., Mohr, L.R., Wood, C. \& Leeton, J.F. (1982) Effect of delayed insemination on in vitro fertilization, culture and transfer of human embryos. $J$. Reprod. Fert. 64, 285-294.

Veeck, L.L., Wortham, J.W.E., Witmyer, J., Sandow, B.A., Acosta, A.A., Garcia, J.E., Jones, G.S. \& Jones, H.W. (1983) Maturation and fertilization of morphologically immature human oocytes in a program of in vitro fertilization. Fert. Steril. 39, 594-602.

Wolf, D.P. (1981) The mammalian egg's block to polyspermy. In Fertilization and Embryonic Development In Vitro, pp. 183-197. Eds L. Mastroianni \& J. D. Biggers. Raven Press, New York.

Zenzes, M.T., Belkien, L., Bordt, J., Kan, I., Schneider, H.P.G. \& Nieschlag, E. (1985) Cytologic investigation of human in vitro fertilization failures. Fert. Steril. 43, 883-891. 